\title{
"Nem Todas as Vítimas Importam": Crenças no Mundo Justo, Relações Intergrupais e Responsabilização de Vítimas
}

\author{
João Gabriel Modesto ${ }^{1}$ \\ Universidade Estadual de Goiás, Goianésia, GO, Brasil \\ Universidade de Brasília, Brasília, DF, Brasil \\ Ronaldo Pilati \\ Universidade de Brasilia, Brasília, DF, Brasil
}

\begin{abstract}
Resumo
A presente pesquisa investigou o processo de responsabilização de vítimas no contexto das relações intergrupais a partir da hipótese do mundo justo. Em dois estudos testamos a influência da categoria social da vítima na relação entre crenças no mundo justo (CMJ) explícita $\backslash$ implícita e a responsabilização de vítimas. No Estudo 1, 102 participantes responderam a medidas explícita e implícita da CMJ e avaliaram o grau de responsabilidade de uma vítima (endogrupo X exogrupo) de bala perdida. No Estudo 2, além da manipulação da categoria social da vítima, incluímos uma manipulação de carga cognitiva. Foram 110 participantes que responderam às medidas da $\mathrm{CMJ}$ e avaliaram a responsabilidade de uma vítima (endogrupo X exogrupo) de bala perdida em uma situação de carga cognitiva (baixa X alta). Verificou-se que a categoria social da vítima interfere na relação da CMJ com a responsabilização. Quando a CMJ explícita ou implícita explicou a responsabilização, esse efeito foi restrito para a avaliação da vítima do endogrupo. Os achados da presente pesquisa corroboram a hipótese que vítimas do endogrupo são mais ameaçadoras para a CMJ dos indivíduos, indicando a importância de considerar a categoria social da vítima para um adequado entendimento da hipótese do mundo justo.
\end{abstract}

Palavras-chave: Crenças no mundo justo, processos implícitos, relações intergrupais, carga cognitiva.

\section{"Not All Victims Matter": Belief in a Just World, Intergroup Relations and Victim Blaming}

\begin{abstract}
The present research investigated the process of victim blaming in the context of intergroup relations through the just world hypothesis. In two studies we tested the influence of the victim's social category in the relationship between explicit and implicit beliefs in a just world (BJW) and victim blaming. In Study 1, 102 participants answered the explicit and implicit measures of BJW and evaluated how much a stray bullet victim (ingroup X outgroup) was to blame. In Study 2, aside from the manipulation of victim social category, we included a manipulation of cognitive load. 110 participants answered the BJW measures and evaluated how much a stray bullet victim (ingroup X outgroup) was to blame in a situation of cognitive load (low X high). It was verified that the victim's social category interferes in the relationship

Endereço para correspondência: Universidade de Brasília, Campus Universitário Darcy Ribeiro, Instituto Central de Ciências, Instituto de Psicologia, Brasília, DF, Brasil 70.910-900. Fone: (71) 98767-8566. E-mail: jg.modesto@gmail.com

O presente trabalho recebeu apoio da CAPES na forma de bolsa de mestrado para o primeiro autor, bem como do CNPq na forma de Bolsa Produtividade em Pesquisa (PQ) e apoio individual no Edital Universal CNPq (478285/2013-2) para o segundo autor
\end{abstract}


between BJW and victim blaming. When explicit or implicit BJW explained blaming, that effect was restricted to the evaluation of an ingroup victim. The findings of the present research confirm the hypothesis that ingroup victims are more threatening to individual's BJW and indicate the importance of considering the victim's social category for a more adequate understanding of the just world hypothesis.

Keywords: Belief in a just world, implicit processes, intergroup relations, cognitive load.

\section{“Ni Todas las Victimas Importan": Creencia en el Mundo justo, Relaciones Intergrupales y Reponsabilizacion de Victimas}

\section{Resumen}

Esta investigación examino' el proceso de responsabilizacion de victimas en el contexto de las relaciones intergrupales a partir de la hipótesis del mundo justo. En dos estudios investigamos la influencia de la categoría social de la víctima en la relación entre creencias en el mundo justo (CMJ) explicita/implícita y la responsabilizacion de victimas. En el Estudio 1, 102 participantes respondieron medidas explicita y implícita de CMJ y evaluaron el grado de responsabilidad de una víctima (endogrupo X exogrupo) de bala perdida. En el Estudio 2, además de la manipulación de categoría social de la víctima, incluimos una manipulación de carga cognitiva. 110 participantes respondieron las medidas de la CMJ y evaluaron la responsabilidad de una víctima (endogrupo X exogrupo) de bala perdida en una situación de carga cognitiva (baja $\mathrm{X}$ alta). Fue encontrado que la categoría social de la víctima interfiere en la relación de la CMJ con la responsabilizacion. Cuando la CMJ explícita o implícita explicó la responsabilizacion, este efecto fue restricto a la evaluación de la víctima del endogrupo. Los resultados de esta investigación confirman la hipótesis de que víctimas del endogrupo son más amenazantes para la CMJ de los individuos y indican la importancia de considerar la categoría social de la victima para una comprensión adecuada de la hipótesis del mundo justo.

Palabras clave: Creencias en el mundo justo, procesos implícitos, relaciones intergrupales, carga cognitiva.

Imagine duas situações. Na primeira delas, um colega de trabalho é vítima de uma bala perdida. Na outra situação, o acidente ocorre com um desconhecido. As duas situações são avaliadas da mesma maneira? A vítima teve responsabilidade no acidente? O que interfere no processo de avaliação de uma situação de vitimização? De acordo com a hipótese do mundo justo (Lerner, 1980), o quanto as pessoas acreditam que o mundo é um lugar justo é um fator que ajuda a explicar a reação a episódios de vitimização. Considerando que outros estudos têm demonstrado a importância da categoria social da vítima para um adequado entendimento da hipótese do mundo justo (Aguiar, Vala, Correia, \& Pereira, 2008; Correia, Alves, Morais, \& Ramos, 2015; Correia et al., 2012; Correia, Vala, \& Aguiar, 2007; Halabi, Statman, \& Dovidio, 2015), na presente pesquisa buscou-se investigar o papel moderador da categoria social da vítima na rela- ção entre as dimensões explícita e implícita das crenças no mundo justo (CMJ) e o processo de responsabilização de vítimas.

\section{Dimensões Implícita e Explícita da CMJ e Reações a Vítimas}

Segundo a compreensão sobre a $\mathrm{CMJ}$, as pessoas agem como se o mundo fosse justo e ordenado, ao aceitarem o entendimento que as pessoas têm o que merecem e merecem o que têm (Lerner, 1980). O confronto com vítimas inocentes se torna, assim, ameaçador por romper com esse senso de justiça (Ramos, Correia, \& Alves, 2014). As pessoas buscam então corrigir a sensação de injustiça, de modo a evitar uma dissonância cognitiva e manter a crença de que o mundo é um lugar justo (Hafer \& Rubel, 2015). Desse modo, muitas vezes, vítimas inocentes acabam sendo responsabilizadas como forma de manutenção da CMJ (Lerner \& Simmons, 1966). 
No entanto, esse processo nem sempre é consciente, acessível pela introspecção (Lerner, 2003), o que tem levado ao entendimento da existência das dimensões explícita e implícita da CMJ (Lerner, 1998; Lerner \& Goldberg, 1999). Para uma melhor compreensão do construto, têm sido então utilizadas estratégias diretas (Dalbert, 1999; Gouveia, Pimentel, Coelho, Maynart, \& Mendonça, 2010; Modesto, Figueredo, Gama, Rodrigues, \& Pilati, 2017; Rubin \& Peplau, 1975) e indiretas para mensuração da CMJ (Callan, Ferguson, \& Bindemann, 2013; Hafer, 2000; Murray, Spadafore, \& McIntosh, 2005).

Em relação às estratégias indiretas, é possível encontrar na literatura tanto medidas situacionais como medidas disposicionais. A principal medida indireta situacional é um teste Stroop adaptado que permite identificar se determinada situação de vitimização gera uma ameaça à manutenção da CMJ (Hafer, 2000). A medida consiste na avaliação de dois grupos de palavras, um com termos relacionados à justiça (e.g. justiça e justo) e outro grupo com palavras neutras (e.g. telefone e madeira). As palavras são apresentadas como um priming subliminar em uma tela escura seguidas por uma máscara (um conjunto de 8 asteriscos) para prevenir o efeito pós-retina. A tarefa consiste em responder a cor da palavra, sendo a variável dependente o tempo para realização da tarefa. A autora identificou que em situações ameaçadoras para a CMJ, o tempo de latência para a identificação da cor das palavras relacionadas à justiça é maior se comparado à identificação da cor das palavras neutras.

Mais recentemente, entendendo a $\mathrm{CMJ}$ como um traço disposicional, foi desenvolvido um Teste de Associação Implícita (TAI; Greenwald, Mcghee, \& Schwartz, 1998) para mensurar a dimensão implícita da CMJ (Modesto \& Pilati, 2015). O TAI avalia, por meio do tempo de resposta ao associar determinados conceitos, a atitude implícita dos participantes. No caso do TAI-CMJ, é possível avaliar a atitude implícita sobre noções ligadas à estabilidade $\backslash$ imprevisibilidade do mundo, conceitos chaves para a compreensão da dimensão não consciente da CMJ (Modesto \& Pilati, 2015 para mais informações sobre o TAI). O TAI se diferencia de estratégias indiretas situacionais por mensurar a CMJ do indivíduo a priori, como um traço disposicional, independente da situação de vitimização avaliada.

\section{CMJ e a Categoria Social de Vítimas}

A despeito das evidências da relação entre CMJ e a responsabilização de vítimas (Hafer \& Bègue, 2005 para uma revisão), não é qualquer situação de vitimização que gera uma ameaça à manutenção da CMJ.

Diferentes vítimas oferecem diferentes ameaças à $\mathrm{CMJ}$ dos indivíduos. Uma vítima inocente, cujo sofrimento persiste, parece ser o tipo mais ameaçador à manutenção da CMJ (Correia \& Vala, 2003; Lerner \& Simmons, 1966). Por outro lado, usualmente as pessoas estão interessadas no que ocorre em "seu próprio mundo", de modo que o confronto com vítimas de outros contextos e grupos parece ser menos ameaçador (Lerner \& Miller, 1978). Estudos têm confirmado essa hipótese tanto por meio de medidas indiretas situacionais (Aguiar et al., 2008; Correia et al., 2007), como através de medidas explícitas (Correia et al., 2012).

Embora uma vítima do endogrupo seja mais ameaçadora à $\mathrm{CMJ}$, isso não implica em uma maior desqualificação e culpabilização da vítima do endogrupo quando comparada a vítimas do exogrupo, prevalecendo um favoritismo endogrupal (Aguiar et al., 2008; Anderson, 1992; Correia et al., 2007; Kleinke \& Meyer, 1990). O confronto com vítimas do exogrupo, sobretudo quando avaliados membros de grupos alvo de preconceito e estereótipos, parece oferecer menos risco à manutenção da $\mathrm{CMJ}$ devido à utilização de mecanismos justificadores (Jost \& Banaji, 1994; Lima-Nunes, Pereira, \& Correia, 2013).

\section{Estudo 1}

Apesar das evidências sobre a importância de considerar a categoria social da vítima para um adequado entendimento dos efeitos da CMJ explícita e implícita, é preciso ressalvar diferenças conceituais e metodológicas entre essas tradições de pesquisa. 
Em estudos sobre a CMJ explícita e relações intergrupais, uma medida direta de CMJ é utilizada, a categoria social da vítima manipulada e então é testado o efeito exercido pela CMJ na reação à vítima considerando a manipulação experimental (Correia et al., 2012). Esses estudos têm indicado que o efeito da $\mathrm{CMJ}$ tende a ocorrer na avaliação de membros do endogrupo, mas não na avaliação de membros do exogrupo.

Em relação aos estudos sobre a CMJ implícita no contexto das relações intergrupais, a CMJ não tem sido medida como um traço, mas sim a partir da avaliação da ameaça à CMJ. Estudos usando o teste Stroop adaptado (Hafer, 2000) têm indicado que o contato com vítimas do endogrupo é mais ameaçador para a CMJ (Correia et al., 2007) e consequentemente o efeito da $\mathrm{CMJ}$ tem sido restrito à avaliação dos membros do endogrupo (Aguiar et al., 2008).

Embora a influência da categoria social da vítima na relação entre $\mathrm{CMJ}$ e reação a vítimas tenha sido testada por meio de medidas diretas e indiretas situacionais, não encontramos na literatura estudos que testem essa relação quando mensurada a CMJ simultaneamente por medidas diretas e indiretas disposicionais. A mensuração, em um mesmo estudo, das dimensões explícita e implícita traz a vantagem de permitir a comparação do efeito dessas variáveis na explicação da reação a vítimas. Feitas essas considerações, o Estudo 1 teve como objetivo testar o papel moderador da categoria social da vítima (endogrupo $\mathrm{X}$ exogrupo) na relação entre CMJ explícita) implícita e a responsabilização de vítimas. A hipótese é que, embora membros do exogrupo sejam mais responsabilizados que membros do endogrupo, apenas quando avaliados membros do endogrupo a CMJ exercerá uma influência positiva na responsabilização da vítima.

\section{Método}

\section{Participantes}

Participaram do estudo 104 estudantes universitários, sendo dois casos excluídos por não terem respondido integralmente à pesquisa. Dos 102 participantes, $51,00 \%$ eram do sexo mascu- lino, com idades variando de 16 a 34 anos $(M=$ $20,45 ; D P=3,18)$.

\section{Instrumentos}

Para avaliação da CMJ explícita, utilizouse a versão traduzida para o português da Escala Global de Crenças no Mundo Justo - EGCMJ (Gouveia et al., 2010; $\alpha=0,77$ ). Os itens da EGCMJ variam de 1 (discordo totalmente) a 6 (concordo totalmente), com maiores valores indicando maiores índices de CMJ explícita.

A dimensão implícita da CMJ foi mensurada através do TAI para dimensão global da CMJ (Modesto \& Pilati, 2015; $\alpha=0,70$ ). O resultado do TAI oscila de $-2 \mathrm{a}+2$, sendo que maiores valores representam maiores índices de CMJ implícita. Optou-se pelo TAI por ser, até onde encontramos na literatura, a única medida indireta disposicional para a CMJ.

Para avaliar a responsabilização da vítima, foram utilizados três itens, em escala de 1 (nada\ de modo algum) a 7 (totalmente $\backslash$ completamente), adaptados de estudos anteriores (Correia, 2003; "X" é responsável pela situação em que se encontra?; " $X$ " é culpado pela situação em que se encontra?; " $\mathrm{X}$ " poderia ter evitado o ocorrido?) que agrupados apresentaram consistência interna satisfatória $(\alpha=0,80)$.

O cenário avaliado pelos participantes foi apresentado como uma reportagem que relatava o caso de uma vítima de bala perdida que se encontrava em estado grave de saúde necessitando realizar uma cirurgia, apresentando assim as características da vítima que mais ameaçam a manutenção da CMJ: uma vítima inocente cujo sofrimento persiste (Correia \& Vala, 2003; Lerner \& Simmons, 1966). Para a manipulação da categoria social da vítima, na condição de endogrupo a vítima era apresentada como estudante universitário, já na condição de exogrupo era apresentada como usuário de crack.

Para garantir a composição do usuário de crack como exogrupo, ao final do estudo havia uma checagem de manipulação experimental em que o participante deveria responder se era usuário de substâncias psicoativas e qual substância 
utilizava. O participante que informasse ser usuário de crack seria excluído da amostra.

\section{Procedimentos de Coleta de Dados}

Estudantes foram abordados no campus de uma universidade e convidados a participar voluntariamente da pesquisa. Os participantes respondiam inicialmente ao TAI e à medida explícita da CMJ. Em seguida, eram alocados aleatoriamente na condição de endogrupo ou exogrupo para avaliação do cenário. Respondiam sobre o grau de responsabilidade da vítima, ao item de checagem de manipulação e, por fim, informavam dados sócio-demográficos. Após a conclusão do estudo, os participantes recebiam o debriefing. Toda a coleta foi feita em laboratório em sessões com até quatro participantes. Cada participante deveria responder individualmente aos instrumentos em um computador. A pesquisa foi desenvolvida no software Inquisit versão 4.0 .

A presente pesquisa não ofereceu riscos de cunho físico, moral ou psicológico, seguindo os princípios éticos de pesquisa com seres humanos postulados pela APA. Todos participantes consentiram a participação, lhes sendo garantido o anonimato das informações prestadas.

\section{Procedimento de Análise de Dados}

Todas as análises foram conduzidas no Software SPSS (Statistical Package for the Social Sciences) versão 18.0. Para a comparação entre as condições experimentais, foram realizadas Análises de Variância (ANOVA). Para os testes do efeito da CMJ na responsabilização de vítimas, foram realizadas regressões lineares. A hipótese de moderação foi testada, conforme recomendações da área (Hayes, 2013), com o uso do PROCESS.

\section{Resultados}

Apenas cinco participantes informaram consumir algum tipo de substância ilícita, sendo que ninguém relatou ser usuário de crack, garantindo assim a composição do usuário de crack como membro do exogrupo, não sendo necessá- rio excluir nenhum caso. O critério de exclusão apenas dos participantes usuários de crack, e não dos usuários de demais tipos de drogas, se justifica porque no Brasil o usuário de crack configura-se como um grupo alvo de estereótipos e preconceito que não necessariamente se aplicam aos usuários de outras drogas. Ainda assim, as análises relatadas a seguir foram conduzidas, em um primeiro momento, com a exclusão dos participantes usuários de outras drogas, apresentando resultados similares.

A fim de testar o efeito direto da manipulação experimental da categoria social da vítima na responsabilização, foi conduzida uma ANOVA em que se verificou que membros do exogrupo $(M=2,73 ; D P=1,50)$ foram mais responsabilizados que membros do endogrupo $(M=1,89, D P=1,21), F(1,100)=9,50, p=$ $0,003, \eta^{2} p=0,09$.

$\mathrm{O}$ efeito direto da CMJ na responsabilização foi testado através de regressões lineares. Não foram encontrados resultados significativos tanto para a dimensão explícita, $\beta=0,17, t(101)$ $=1,73, p=0,087$, como para dimensão implícita, $\beta=0,13, t(101)=1,28, p=0,203$.

Sobre o teste de moderação, para a CMJ explícita, embora a interação entre CMJ e categoria social da vítima não tenha sido significativa, $\beta$ $=0,30, t(101)=1,56, p=0,122$, não caracterizando uma moderação, verifica-se que apenas na avaliação de um membro do endogrupo a $\mathrm{CMJ}$ prediz a responsabilização, $\beta=0,52, t(101)=$ $2,50, p=0,014$, não sendo significativo o efeito quando avaliado um membro do exogrupo, $\beta=$ $0,01, t(101)=0,05, p=0,963$. Para a dimensão implícita, não foi encontrado nenhum efeito na avaliação da vítima.

\section{Discussão}

O Estudo 1 investigou o papel moderador da categoria social da vítima na relação entre CMJ explícita implícita e a responsabilização de vítimas. A hipótese afirmava que, embora membros do exogrupo fossem mais responsabilizados, apenas quando avaliados membros do endogrupo a CMJ exerceria uma influência na responsabilização da vítima. Como esperado, o membro 
do exogrupo foi mais responsabilizado que o membro do endogrupo, confirmando o entendimento de um favoritismo endogrupal encontrado em estudos anteriores sobre a responsabilização de vítimas (Aguiar et al., 2008; Anderson, 1992; Correia et al., 2007; Kleinke \& Meyer, 1990). A atribuição de responsabilidade para uma vítima do exogrupo, sobretudo para membros de grupos alvo de preconceitos e estereótipos, representa uma forma de manutenção do status quo através de mecanismos justificadores (Jost \& Banaji, 1994; Lima-Nunes et al., 2013). Em última instância, esse raciocínio se coaduna com a hipótese do mundo justo, ao assumir que coisas boas acontecem a pessoas boas e coisas ruins acontecem a pessoas ruins (Lerner, 1998). Desse modo, vítimas de grupos alvo de estereótipos e preconceito, como é o caso do usuário de crack no Brasil, tendem a ser mais responsabilizadas quando comparados a vítimas do endogrupo.

Sobre a influência da CMJ, a hipótese foi parcialmente corroborada. Embora o resultado da moderação não tenha sido estatisticamente significativo, a CMJ influenciou a responsabilização apenas de vítimas do endogrupo, confirmando o entendimento que as pessoas estão interessadas no que ocorre "em seu próprio mundo", e que vítimas de outros contextos e grupos não são tão ameaçadoras à manutenção da CMJ (Lerner \& Miller, 1978). Esse resultado pode ser interpretado como consequência de perceber membros do exogrupo como fora das fronteiras da justiça (Lima-Nunes et al., 2013; Opotow, 1990). Desse modo, situações de injustiça com esses grupos não tendem a ameaçar a CMJ dos indivíduos.

O Estudo 1 apresentou algumas limitações. $\mathrm{O}$ efeito da $\mathrm{CMJ}$ se limitou à dimensão explícita, não tendo sido encontrado qualquer efeito da dimensão implícita. É possível que esse resultado tenha sido influenciado pelo tipo de cenário avaliado. Foi utilizado um cenário deliberativo, em que os participantes dispunham de amplos recursos cognitivos. Nessas situações, as medidas explícitas costumam ser melhores preditores que as medidas implícitas (Asendorpf, Banse, \& Mücke, 2002; Fleischhauer, Strobel, Enge, \& Strobel, 2013). Esse entendimento, somado aos resultados do Estudo 1, apontou para a necessidade de testar as hipóteses também em cenários menos deliberativos.

\section{Estudo 2}

Considerando os achados do Estudo 1, e o entendimento que em cenários deliberativos medidas explícitas costumam ser melhores preditores que medidas implícitas, o Estudo 2 teve como objetivo testar o papel moderador da categoria social da vítima na relação entre $\mathrm{CMJ}$ e responsabilização em cenários distintos: alta e baixa carga cognitiva. Carga cognitiva pode ser definida como a restrição de recursos cognitivos para processar determinada quantidade de informação (Dijksterhuis \& Van Knippenberg, 1995). A manipulação da carga cognitiva foi proposta para criar cenários menos deliberativos. Outros estudos indicam que nessas situações medidas implícitas possuem maior poder preditivo que medidas explícitas (Fleischhauer et al., 2013).

O Estudo 2 tem, então, um desenho experimental entre participantes: 2 (categoria social da vítima: endogrupo e exogrupo) X 2 (carga cognitiva: alta e baixa). As hipóteses para o Estudo 2 permanecem as mesmas: embora membros do exogrupo serão mais responsabilizados que membros do endogrupo, apenas quando avaliado o endogrupo será encontrada uma relação positiva entre CMJ e responsabilização. Adicionalmente, presume-se que na condição de carga alta, o efeito da CMJ implícita será mais robusto que o da CMJ explícita enquanto que na condição de carga baixa o efeito da CMJ explícita irá prevalecer se comparado à CMJ implícita.

\section{Método}

\section{Participantes}

Participaram do estudo 115 estudantes universitários, no entanto, cinco casos foram excluídos por não terem completado o procedimento. Dos 110 casos válidos, foram $56,4 \%$ do sexo feminino e $43,6 \%$ do sexo masculino com idades variando de 18 a 55 anos $(M=24,01$; $D P=6,29)$. 


\section{Instrumentos}

Assim como no Estudo 1, utilizou-se a versão traduzida para o português da EGCMJ para mensurar a CMJ explícita $(\alpha=0,85)$, o TAI para a CMJ implícita $(\alpha=0,58)$ e os mesmo três itens para avaliar a responsabilização da vítima $(\alpha=$ $0,84)$.

O cenário para o Estudo 2, assim como no Estudo 1, era apresentado como uma reportagem que relatava o caso de uma vítima de bala perdida que se encontrava em estado grave de saúde e necessitava realizar uma cirurgia. No entanto, para facilitar a manipulação de carga, o cenário foi estendido sendo apresentado em 11 vinhetas. Para o estabelecimento da carga cognitiva, restringiu-se o tempo de leitura do cenário. O participante, na condição de carga alta, dispunha de 7 segundos para ler cada vinheta. Para a condição de baixa carga, o tempo de leitura era livre. Essa manipulação foi baseada em estudos anteriores (Van Knippenberg, Dijksterhuis, \& Vermeulen, 1999) e parte do conceito de carga cognitiva como restrição de recursos cognitivos para processar determinada quantidade de informação (Dijksterhuis \& Van Knippenberg, 1995). Desse modo, ao restringir o tempo, os participantes estarão em uma situação de maior carga cognitiva do que os participantes na condição de tempo ilimitado.

Do total de 11 vinhetas, as quatro primeiras serviam para ativar a categoria social da vítima, bem como apresenta-la como uma vítima inocente cujo sofrimento persiste. As demais vinhetas apresentavam maiores detalhes sobre o episódio (e.g. "X esteve com a(o) namorada(o) pouco antes do ocorrido. 'Estava comigo antes de ir. É difícil de acreditar. Tínhamos, inclusive, combinado de nos encontrar quando $[\mathrm{X}]$ voltasse da casa do amigo. Agora só me resta aguardar'”). A quantidade de palavras por vinheta variou de 34 a 36 . No entanto, para as quatro primeiras vinhetas, optou-se por um número menor de palavras (de 17 a 27 palavras) para uma maior garantia que as informações sobre a categoria social da vítima, sua inocência e gravidade da situação fossem processadas de maneira mais deliberada mesmo na condição de carga alta. Essa decisão foi tomada para aumentar a chance de uma maior efetividade da manipulação experimental e da ativação das características da vítima que são mais ameaçadoras para a CMJ. A decisão pela quantidade de vinhetas, o tempo de leitura e o número de palavras por vinheta foi tomada com base em estudos anteriores (Van Knippenberg et al., 1999) e na realização de pré-testes.

Para a checagem da manipulação da carga, o participante deveria responder a um item sobre a pressão do tempo ao ler a notícia ("Você se sentiu pressionado pelo tempo ao ler a reportagem?") que variava de 1 (discordo totalmente) a 10 (concordo totalmente).

\section{Procedimentos de Coleta de Dados}

Assim como no Estudo 1, os participantes foram estudantes universitários abordados no campus da universidade. Inicialmente respondiam ao TAI-CMJ e à medida explícita. Em seguida, deveriam ler o cenário, responder à checagem da manipulação de carga, aos itens de responsabilização e informar dados sócio demográficos. A coleta foi realizada em laboratório em sessões com até quatro participantes. Cada participante deveria responder individualmente aos instrumentos em um computador. A pesquisa foi desenvolvida no software Inquisit versão 4.0. O Estudo 2 seguiu os mesmos cuidados éticos como descrito no Estudo 1.

\section{Procedimentos de Análise de Dados}

Assim como no Estudo 1, as análises foram realizadas no SPSS versão 18.0. Novamente, as comparações entre grupos foram feitas através da ANOVA, os efeitos diretos na responsabilização através de regressões lineares, e o teste de moderação com o auxílio do PROCESS.

\section{Resultados}

Do total de participantes, oito afirmaram consumir algum tipo de substância ilícita, sendo que nenhum relatou ser usuário de crack, permitindo assim considerar a categoria de usuário de crack como exogrupo. Assim como no Estudo 1, as análises foram conduzidas, em um primeiro momento, excluindo os participantes usuários de 
outras drogas e novamente foram encontrados resultados similares.

Sobre a manipulação da carga, a partir da ANOVA, verificou-se que os participantes na condição de carga alta sentiram-se mais pressionados pelo tempo $(M=7,39 ; D P=2,60)$ do que os participantes na condição de carga baixa $(M=$ $5,04 ; D P=2,41), F(1,108)=24,29, p<0,001$, $\eta^{2} p=0,18$, o que indica que o tempo utilizado gerou uma carga cognitiva.

Assim como no Estudo 1, membros do exogrupo $(M=2,62 ; D P=1,50)$ foram mais responsabilizados que membros do endogrupo $(M=$ $1,80, D P=0,87), F(1,108)=12,49, p=0,001$, $\eta^{2} p=0,10$. Esse efeito foi independente da carga cognitiva como pode ser visualizado na Figura 1.

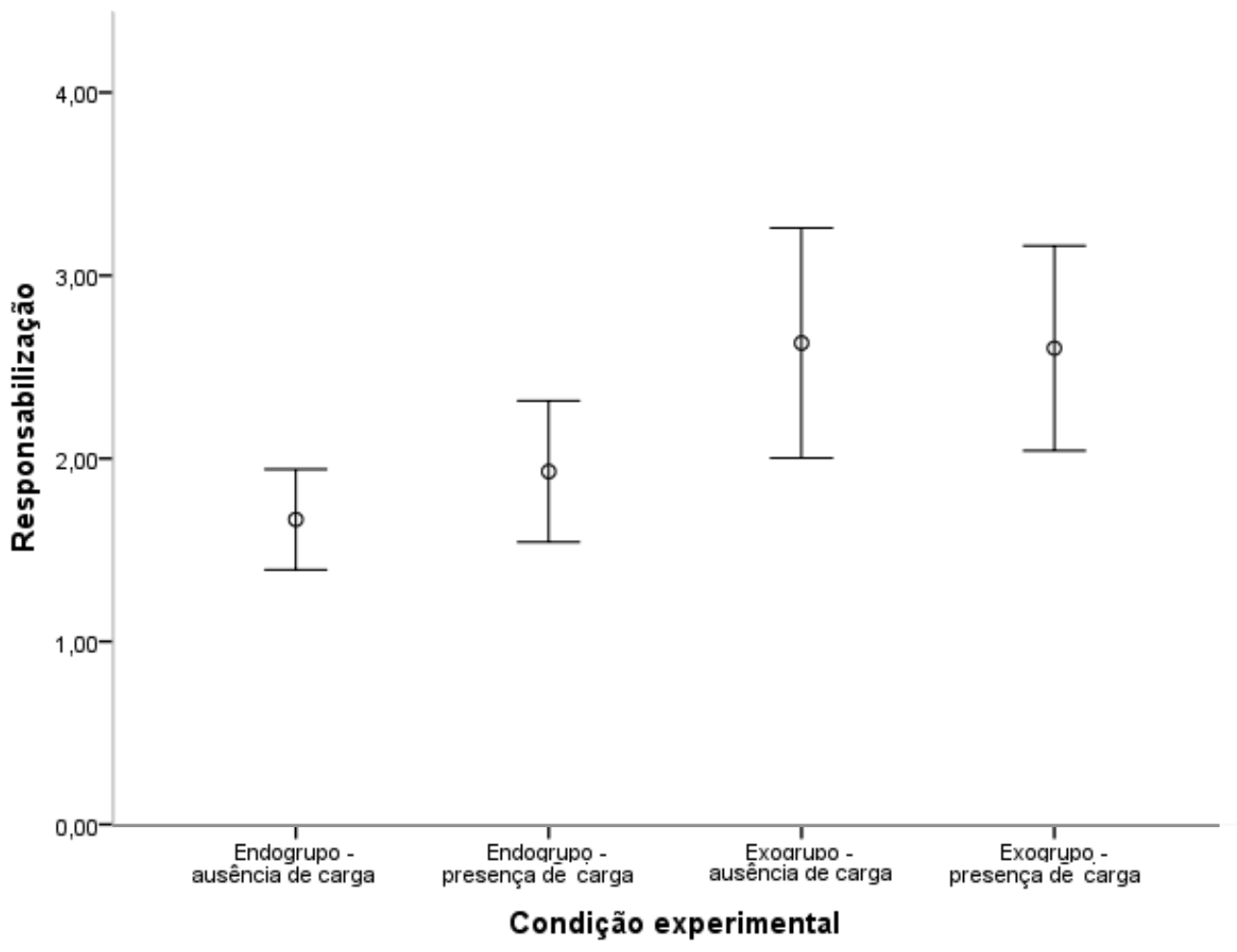

Error Bars: 95\% Cl

Figura 1. Diagrama de barras de erro do nível de responsabilização por condição experimental

Em relação ao efeito direto da $\mathrm{CMJ}$, a partir de regressões lineares, verificou-se que a CMJ explícita explicou $6,5 \%$ da variância da responsabilização, $\beta=0,26, t(109)=2,74, p=0,007$. No entanto, a CMJ implícita não contribuiu significativamente com a explicação direta da responsabilização, $\beta=0,10, t(109)=1,09, p=$ 0,279 . A manipulação da carga não influenciou a responsabilização da vítima, $F(1,108)=0,18$, $p=0,67$.

Testando o papel moderador da categoria social da vítima na relação entre CMJ e responsabilização, considerando apenas a condição de carga baixa, encontra-se uma interação entre
CMJ explícita e categoria social, $\beta=0,83, t$ (54) $=3,68, p<0,001$, com a CMJ explícita explicando a responsabilização apenas quando avaliados membros do endogrupo, $\beta=0,85, t(54)=4,78, p$ $<0,001$, não sendo significativo o efeito quando avaliados membros do exogrupo, $\beta=0,03, t(54)$ $=0,19, p=0,854$, caracterizando uma moderação. Quando avaliada a condição de carga alta, não é mais encontrado um efeito de interação, $\beta=0,13, t(53)=0,47, p=0,644$. Na condição de carga alta, a CMJ explícita não exerceu influência nem para o endogrupo, $\beta=0,30, t$ (53) $=1,41, p=0,165$, nem para o exogrupo, $\beta=$ $0,17, t(53)=1,01, p=0,316$. 
Para a CMJ implícita, na condição de baixa carga, embora não tenha sido encontrada uma interação, $\beta=0,29, t(55)=1,18, p=0,243$, não caracterizando uma moderação, a CMJ implícita explicou a responsabilização do endogrupo, $\beta=$ $0,39, t(55)=2,11, p=0,040$, não sendo significativo o efeito para o exogrupo, $\beta=0,10, t(55)=$ $0,61, p=0,547$. Já na condição de carga alta, não foi encontrado qualquer efeito de interação, $\beta=$ $0,11, t(53)=0,26, p=0,797$. Para essa condição, a CMJ implícita não influenciou a responsabilização do endogrupo, $\beta=0,07, t(53)=0,39, p$ $=0,702$, nem do exogrupo, $\beta=0,01, t(53)=$ $0,01, p=0,991$.

\section{Discussão}

O Estudo 2 testou a hipótese de moderação da categoria social da vítima na relação entre CMJ explícita\implícita e a responsabilização de vítimas em cenários distintos: carga cognitiva baixa e alta.

Assim como no Estudo 1, a hipótese que vítimas do exogrupo seriam mais responsabilizadas que vítimas do endogrupo foi corroborada. Os achados do Estudo 2 tendem a reafirmar o entendimento de um favoritismo endogrupal encontrado em outros estudos sobre a responsabilização de vítimas (Aguiar et al., 2008; Correia et al., 2012; Correia et al., 2007).

Sobre a CMJ explícita, foi corroborada a hipótese de moderação da categoria social da vítima na explicação da responsabilização de vítimas. A CMJ explícita contribuiu com a explicação da responsabilização de vítimas apenas do endogrupo, confirmando o entendimento que vítimas do endogrupo são mais ameaçadoras para a manutenção da CMJ que vítimas do exogrupo (Lerner \& Miller, 1978). O efeito de interação da CMJ explícita e categoria social da vítima foi encontrado apenas em um cenário mais deliberativo, em que os participantes dispunham de mais recursos cognitivos para avaliar o cenário. Na situação de carga cognitiva alta (cenário menos deliberativo) não foi encontrado um efeito direto da CMJ explícita e nem a interação entre $\mathrm{CMJ}$ e categoria social da vítima. Considerando que medidas explícitas tendem a apresentar melhor poder explicativo quando o cenário avaliado permite a utilização de mais recursos cognitivos (Asendorpf et al., 2002; Fleischhauer et al., 2013), era esperado que o efeito da CMJ explícita se limitasse ao cenário mais deliberativo.

Para a CMJ implícita, a hipótese foi parcialmente corroborada. Embora o teste da moderação não tenha sido estatisticamente significativo, a CMJ implícita explicou a responsabilização da vítima do endogrupo, mas não a do exogrupo. Esse achado reafirma a importância de considerar a categoria social da vítima nas investigações sobre a CMJ, inclusive quando acessada através de medidas indiretas (Aguiar et al., 2008; Correia et al., 2007).

$\mathrm{O}$ efeito encontrado para a CMJ implícita, no entanto, se limitou ao cenário com baixa carga cognitiva, diferente da hipótese formulada. Esperava-se que a manipulação da carga cognitiva, através da restrição do tempo para a leitura do cenário, gerasse um cenário menos deliberativo, o que facilitaria a expressão do efeito da CMJ implícita e consequentemente sua interação com a categoria social da vítima na explicação da responsabilização. No entanto, não houve efeito na condição de carga alta e sim na condição de baixa carga. Uma hipótese alternativa é que embora pré-testes tenham sido conduzidos, o tempo utilizado para gerar a carga foi muito baixo e os participantes não foram capazes de processar as informações do cenário.

Mas o que levou a um resultado significativo na condição de baixa carga? Há evidências que fatores estressores prejudicam a capacidade cognitiva dos indivíduos (Klein \& Boals, 2001), como, por exemplo, em situações de ameaça (Schmader \& Johns, 2003). Nessas situações, os indivíduos tendem a direcionar recursos cognitivos para suprimir pensamentos e sentimentos negativos não desejados e, consequentemente, tendem a se ocupar menos com tarefas que estejam realizando (Schmader \& Johns, 2003). Considerando que situações de vitimização geram uma ameaça à $\mathrm{CMJ}$, o cenário apresentado pode ter afetado a capacidade cognitiva dos par- 
ticipantes, o que levou a um efeito significativo da CMJ implícita no cenário com baixa carga. No Estudo 1, em um cenário breve, não houve efeito da dimensão implícita. Já no Estudo 2, em um cenário com um conjunto maior de informações, demandando maior atenção para processar a informação, a apresentação da ameaça por si só pode ter gerado a diminuição de recursos cognitivos disponíveis, o que permitiu um efeito da dimensão implícita da CMJ.

\section{Discussão Geral}

A presente pesquisa teve como objetivo testar o papel moderador da categoria social da vítima na relação entre CMJ explícita implícita e a responsabilização de vítimas. Foram conduzidos dois estudos em que se verificou que nas situações em que a CMJ explícita e implícita explicaram a responsabilização, o efeito se restringiu à avaliação de membros do endogrupo. Os achados da presente pesquisa corroboram a hipótese que vítimas do endogrupo são mais ameaçadoras para a CMJ dos indivíduos e reafirmam, portanto, a importância de considerar a categoria social da vítima para um adequado entendimento da hipótese do mundo justo. Esse efeito restrito a membros do endogrupo pode ser interpretado pelo entendimento que membros de grupos alvo de estereótipos e preconceito podem ser percebidos como fora do escopo da justiça (Lima-Nunes et al., 2013; Opotow, 1990). Desse modo, injustiças que ocorrem com esses membros não ameaçam a CMJ dos indivíduos.

Destaca-se que não encontramos na literatura outros estudos que tenham testado o efeito da CMJ na responsabilização de vítimas do endogrupo e exogrupo quando acessada simultaneamente por medidas disposicionais direta e indireta. Os dois estudos apresentados permitiram a comparação dos efeitos das duas dimensões da CMJ. Recomenda-se que novos estudos testem sob quais condições cada uma das dimensões exerce influência na responsabilização de vítimas. É preciso esclarecer, por exemplo, porque a dimensão implícita apresentou efeito apenas na condição de baixa carga. Apresentamos uma hipótese post-hoc que deve ser testada em outros experimentos para uma melhor compreensão do fenômeno.

\section{Referências}

Aguiar, P., Vala, J., Correia, I., \& Pereira, C. (2008). Justice in our world and in that of others: Belief in a Just World and reactions to victims. Social Justice Research, 21(1), 50-68. doi:10.1007/ s11211-007-0059-3

Anderson, V. N. (1992). For whom is this world just?: Sexual orientation and AIDS. Journal of Applied Social Psychology, 22(3), 248-259. doi:10.1111/j.1559-1816.1992.tb01538.x

Asendorpf, J. B., Banse, R., \& Mücke, D. (2002). Double dissociation between implicit and explicit personality self-concept: The case of shy behavior. Journal of Personality and Social Psychology, 83(2), 380-393. doi:10.1037//00223514.83.2.380

Callan, M. J., Ferguson, H. J., \& Bindemann, M. (2013). Eye movements to audiovisual scenes reveal expectations of a just world. Journal of Experimental Psychology. General, 142(1), 3440. doi:10.1037/a0028261

Correia, I. (2003). Concertos e desconcertos na procura de um mundo concertado: Crença no Mundo Justo, inocência da vítima e vitimização secundária. Lisboa: Fundação Calouste Gulbenkian.

Correia, I., Alves, H., Morais, R., \& Ramos, M. (2015). The legitimation of wife abuse among women: The impact of belief in a just world and gender identification. Personality and Individual Differences, 76, 7-12. doi:10.1016/j. paid.2014.11.041

Correia, I., Alves, H., Sutton, R., Ramos, M., Gouveia-Pereira, M., \& Vala, J. (2012). When do people derogate or psychologically distance themselves from victims? Belief in a just world and ingroup identification. Personality and Individual Differences, 53(6), 747-752. doi:10.1016/j. paid.2012.05.032

Correia, I., \& Vala, J. (2003). Crença no mundo justo e vitimização secundária : O papel moderador da inocência da vítima e da persistência do sofrimento. Análise Psicológica, 3(XXI), 341-352. doi:10.14417/ap. 15 
Correia, I., Vala, J., \& Aguiar, P. (2007). Victim's innocence, social categorization, and the threat to the belief in a just world. Journal of Experimental Social Psychology, 43(1), 31-38. doi:10.1016/j.jesp.2005.12.010

Dalbert, C. (1999). The world is more just for me than generally: About the personal belief in a just world scale's validity. Social Justice Research, 12(2), 79-98. doi:10.1023/A:1022091609047

Dijksterhuis, A. P., \& Van Knippenberg, A. (1995). Timing of schema activation and memory: Inhibited access to inconsistent information. European Journal of Social Psychology, 25, 383-390. doi:10.1002/ejsp.2420250403

Fleischhauer, M., Strobel, A., Enge, S., \& Strobel, A. (2013). Assessing implicit cognitive motivation : Developing and testing an Implicit Association Test to measure Need for Cognition. European Journal of Personality, 29(1), 15-29. doi:10.1002/per.1841

Gouveia, V. V., Pimentel, C. E., Coelho, J. A. P. de M., Maynart, V. A. P., \& Mendonça, T. dos S. (2010). Validade fatorial confirmatória e consistência interna da Escala Global de Crenças no Mundo Justo - GJWS. Interação em Psicologia, 14(1), 21-29. doi:10.5380/psi.v14i1.12687

Greenwald, A. G., Mcghee, D. E., \& Schwartz, J. L. K. (1998). Measuring individual differences in implicit cognition: The Implicit Association Test. Journal of Personality and Social Psychology, 74(6), 1464-1480. doi:10.1037/00223514.74.6.1464

Hafer, C. L. (2000). Do innocent victims threaten the belief in a just world? Evidence from a modified Stroop Task. Journal of Personality and Social Psychology, 79(2), 165-173. doi:10.1037/ AW22-3514.79.2.165

Hafer, C. L., \& Bègue, L. (2005). Experimental research on just-world theory: Problems, developments, and future challenges. Psychological Bulletin, 131(1), 128-167. doi:10.1037/0033-2909.131.1.128

Hafer, C. L. \& Rubel, A. N. (2015). The why and how of defending belief in a just world. In J. M. Olson \& M. P. Zanna (Eds.), Advances in Experimental Social Psychology (pp. 41-96). doi:10.1016/ bs.aesp.2014.09.001

Halabi, S., Statman, Y., \& Dovidio, J. F. (2015). Attributions of responsibility and punishment for ingroup and outgroup members:
The role of just world beliefs. Group Processes \& Intergroup Relations, 18(1), 104-115. doi:10.1177/1368430214546067

Hayes, A. F. (2013). Introduction to mediation, moderation, and conditional process analysis: $A$ regression-based approach. New York: The Guilford Press.

Jost, J. T., \& Banaji, M. R. (1994). The role of stereotyping in system-justification and the production of false consciousness. British Journal of Social Psychology, 33(1), 1-27. doi:10.1111/j.2044-8309.1994.tb01008.x

Klein, K., \& Boals, A. (2001). The relationship of life event stress and working memory capacity. Applied Cognitive Psychology, 15(5), 565-579. doi:10.1002/acp.727

Kleinke, C. L., \& Meyer, C. (1990). Evaluation of rape victim by men and women with high and low belief in a just world. Psychology of Women Quarterly, 14(3), 343-353. doi:10.1111/j.1471-6402.1990.tb00024.x

Lerner, M. J. (1980). The Belief in a Just World: A Fundamental Delusion. New York: Plennum Press.

Lerner, M. J. (1998). The two forms of belief in a just world: Some thoughts on why and how people care about justice. In L. Montada \& M. J. Lerner (Eds.), Responses to victimizations and belief in a just world (pp. 247-270). New York: Plenum Press.

Lerner, M. J. (2003). The Justice Motive: Where social psychologists found it, how they lost it, and why they may not find it again. Personality and Social Psychology Review, 7(4), 388-399. doi:10.1207/S15327957PSPR0704_10

Lerner, M. J., \& Goldberg, J. H. (1999). When do decent people blame victims? The differing effects of the explicit/rational and implicit/experiential cognitive systems. In S. Chaiken \& Y. Trope (Eds.), Dual process theories in social psychology (pp. 627-640). New York: Guilford.

Lerner, M. J., \& Miller, D. T. (1978). Just world research and the attribution process: Looking back and ahead. Psychological Bulletin, 85(5), 1030-1051. doi:10.1037//0033-2909.85.5.1030

Lerner, M. J., \& Simmons, C. H. (1966). The observer's reaction to the "innocent victim": Compassion or rejection? Journal of Personality and Social Psychology, 4(2), 203-210. doi:10.1037/h0023562 
Lima-Nunes, A., Pereira, C. R., \& Correia, I. (2013). Restricting the scope of justice to justify discrimination : The role played by justice perceptions in discrimination against immigrants. European Journal of Social Psychology, 43, 627-636. doi:10.1002/ejsp.1981

Modesto, J. G., Figueredo, V., Gama, G., Rodrigues, M., \& Pilati, R. (2017). Escala Pessoal de Crenças no Mundo Justo: Adaptação e evidências de validade. Psico-USF, 22(1), 13-22. doi: 10.1590/1413-82712017220102.

Modesto, J. G., \& Pilati, R. (2015). Implicit Deservingness: Implicit Association Test for Belief in a Just World. Interamerican Journal of Psychology, 49(2), 203-212.

Murray, J. D., Spadafore, J. A., \& McIntosh, W. D. (2005). Belief in a Just World and social perception: Evidence from automatic activation. The Journal of Social Psychology, 145(1), 35-47. doi:10.3200/SOCP.145.1.35-48

Opotow, S. (1990). Moral exclusion and injustice: An introduction. Journal of Social Issues, 46(1), 1-20. doi:10.1111/j.1540-4560.1990.tb00268.x

Ramos, M. R., Correia, I., \& Alves, H. (2014). To believe or not to believe in a just world? The psychological costs of threats to the belief in a just world and the role of attributions. Self and Identity, 13(3), 257-273. doi:10.1080/15298868 .2013 .798890
Rubin, Z., \& Peplau, L. A. (1975). Who believes in a just world? Journal of Social Issues, 31(3), 6589. doi:10.1111/j.1540-4560.1975.tb00997.x

Schmader, T., \& Johns, M. (2003). Converging evidence that stereotype threat reduces working memory capacity. Journal of Personality and Social Psychology, 85(3), 440-52. doi:10.1037/0022-3514.85.3.440

Testé, B., \& Perrin, S. (2013). The impact of endorsing the belief in a just world on social judgments: The social utility and social desirability of Just-World Beliefs for self and others. Social Psychology, 44(3), 209-218. doi:10.1027/18649335/a000105

Van Knippenberg, A., Dijksterhuis, A., \& Vermeulen, D. (1999). Judgement and memory of a criminal act: The effects of stereotypes and cognitive load. European Journal of Social Psychology, 29(2-3), 191-201. doi:10.1002/ (SICI) 1099-0992(199903/05)29:2/3<191::AID-EJSP923>3.0.CO;2-O
Recebido: 22/12/2015

$1^{a}$ revisão: 25/04/2016

Aceite final: 28/04/2016 